\title{
Soft Bone - Hard Arteries: A Link?
}

\author{
Gérard M. London
}

Service de Néphrologie, Centre Hospitalier Manhès, Fleury Mérogis, and INSERM U970, Hôpital Européen

Georges Pompidou, Paris, France

\section{Key Words}

Chronic kidney disease · Bone disorders · Extraosseous and cardiovascular calcifications

\begin{abstract}
Chronic kidney disease is characterized by mineral and various bone disorders associated with extraosseous and cardiovascular calcifications. Experimental studies and clinical observations in the general population and in chronic kidney disease patients show an inverse relationship between the extent of cardiovascular calcifications and bone mineral density or bone metabolic activity. Arterial calcification and osteoporosis are frequently observed in the same subjects and progress in parallel in postmenopausal women, and associations between histomorphometric indices of bone activity and vascular calcifications were also observed in patients with chronic and end-stage kidney diseases. The biological linkage between vascular calcifications and bone changes is certainly a part of the aging process, but in many studies these bone-vascular associations remained significant after adjustment for age, which suggests an age-independent causal relationship. Based on clinical and experimental evidence showing an association between bone disorders and functional and structural changes of the arterial system the concept of a bone-vascular axis was established complementary to the classical kidney-bone axis. Nevertheless, the factors or mechanisms accounting for these associations are not well understood, and could result from (1)
\end{abstract}

arterial disease responsible for bone abnormalities; (2) action of common dysmetabolic or 'toxic' factors and mechanisms acting on bones and vessels, or (3) direct or indirect influence of bone cells and metabolism on the arterial system. This short review aims to illustrate these possible mechanisms.

Copyright $\odot 2011$ S. Karger AG, Base

Arterial and heart valve calcifications are commonly observed with aging and several clinical conditions such as diabetes or chronic kidney disease (CKD) and endstage renal disease (ESRD) [1]. The extent of cardiovascular calcifications in the general population, diabetic patients and kidney patients predicts subsequent cardiovascular mortalityandall-cause mortalitybeyond established conventional risk factors [2]. Arterial calcifications develop in two distinct sites: the intima and media layers of the large and medium-sized arterial wall. Intimal calcifications are found in atherosclerotic plaques, and are associated with common atherosclerotic complications like angina pectoris, myocardial infarction or peripheral artery disease, as well as complications due to abnormal conduit function (arterial stenosis or occlusions). Medial calcifications are frequently observed with age, diabetes and CKD. They are associated with arterial stiffening characterized by increased systolic and decreased diastolic pressure. These functional changes are largely contributing to myocardial ischemia, cardiac hypertrophy

\section{KARGER \\ Fax +4161306 1234 \\ E-Mail karger@karger.ch}

www.karger.com
(C) 2011 S. Karger AG, Basel

$1420-4096 / 11 / 0344-0203 \$ 38.00 / 0$

Accessible online at:

www.karger.com/kbr
Dr. Gérard London

Centre Hospitalier Manhès

8 , rue Roger Clavier

FR-91712 Fleury-Mérogis Cedex (France)

Tel. +33 16925 6485, E-Mail glondon@ club-internet.fr 
and myocardial dysfunctions [3]. Considered as a passive process associated with cellular aging and death, recent studies have shown that arterial (and valvular) calcification is an active process similar to bone formation that is regulated by a variety of genes and proteins involved in bone metabolism $[4,5]$. The calcification results from imbalance between local or systemic promoters and inhibitors with plasma constituents maintaining minerals in solution and inhibiting their deposition in tissues. This process involves transdifferentiation of vascular smooth muscle cells or pericytes into phenotypically distinct, osteoblast-like cells [6].

There is growing clinical and experimental evidence linking bone activity and/or bone mineral density and arterial calcifications or functional abnormalities of the arterial tree. Arterial calcification and osteoporosis are frequently observed in the same subjects. Numerous population-based longitudinal studies have demonstrated associations between osteoporosis and arterial calcifications or arterial stiffness [7-10]. A longitudinal population-based study has shown an association between the progression of aortic calcifications and decreased bone mineral density [7]. The same study demonstrated that the risk of vertebral or hip fracture was significantly higher in women with aortic calcifications [7]. The associations between bone mineral density or histomorphometric indices of bone activity and vascular calcifications and arterial stiffness were also observed in patients with CKD and ESRD [11-13]. An inverse correlation between coronary artery calcification score and vertebral bone mass was observed in dialysis patients [11, 12]. A high systemic arterial calcification score associated with bone histomorphometry suggestive of low bone activity was observed in hemodialysis patients [13, 14]. A significant association between arterial stiffening and low spine bone mineral density or calcaneal osteopenia was observed in CKD and hemodialysis patients [15-17]. While coronary calcification seemed more likely to be associated with low bone volumes $[11,12]$, aortic calcification is associated with indices of bone turnover, increasing in the presence of low bone turnover conditions.

The biological linkage between vascular calcifications and bone changes is certainly a part of the aging process, but in many studies these bone-vascular associations remained significant after adjustment for age, which suggests an age-independent causal relationship [7, 8]. Nevertheless, the factors or mechanisms accounting for these associations are not well understood, and could result from several possibilities: (1) arterial disease responsible for bone abnormalities; (2) action of common dysmetabolic or 'toxic' factors and mechanisms acting on bones and vessels, or (3) direct or indirect influence of bone cells and metabolism on the arterial system.

\section{Arteries as the 'Culprits' of Bone Alterations}

Ischemia resulting from intraosseous atherosclerosis could account for the association between osteoporosis and decreased bone mineral density and arterial calcification. Generalized atherosclerosis also affects bone circulation, and with aging the bone arteries and arterioles are subject to arteriosclerosis with reduction in bone marrow medullary blood supply [18]. Human bone marrow atherosclerotic lesions appear earlier in femoral bone marrow blood supply than in the arteries of the brain or heart [19]. During senescence bone marrow becomes ischemic with diminished medullary blood supply to the cortex, whose blood supply is replaced by periosteal circulation (fig. 1) [18]. These age-associated changes in blood supply develop in parallel with osteoporosis $[20,21]$. That a link between compromised intraosseous circulation and consequent osteoporosis may exist is also suggested by the observation linking decreased bone mineral density with peripheral artery disease [22]. In healthy women, a recent study showed that bone perfusion indices were reduced in subjects with osteoporosis compared with women with osteopenia or normal bone mineral density [21]. Generalized atherosclerosis could account for the bone-vascular calcification link, but this link is usually associated with age. Epidemiological evidence shows that the correlations between bone pathology and cardiovascular events are mostly age-independent. These observations suggest common underlying mechanisms, but the nature and mechanisms responsible for bone-vascular interactions are not well understood.

\section{Common ‘Dysmetabolic' Factors Associated with Bone-Arterial Interaction}

Clinical data show that osteoporosis and vascular or valvular calcifications are influenced by several common risk factors such as diabetes, dyslipidemia, oxidative stress, and advanced glycation end-product accumulation [23-27]. These risk factors have in common that they all converge to the final pathway, i.e., inflammation. Recent experimental studies have provided direct in vivo 
Fig. 1. Blood supply to human femoral diaphysis in youth and senescence [18]. a 42 years, robust medullary arteries that supply the cortex. b 65 years, dominant periosteal arterial supply with ischemic marrow and bone resorptive lacunae. Reproduced with permission of Blackwell Publishing Ltd. [18].
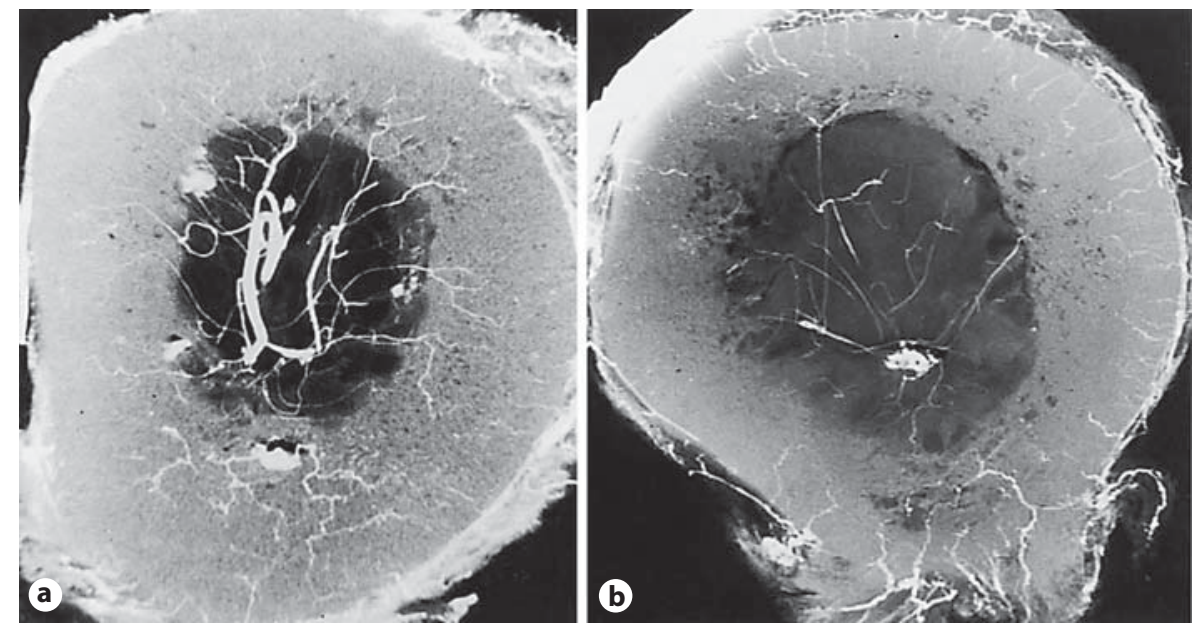

evidence that inflammation inversely correlates with arterial and aortic valve calcification and osteoporotic bone remodeling [28]. Molecular imaging in vivo has demonstrated inflammation-associated arterial osteogenesis in early stages of atherosclerosis [29]. Macrophage activation releases proinflammatory cy tokines (IL- 6 and TNF$\alpha$ ), and proteolytic enzymes with activation of elastolysis and degradation of extracellular matrix metalloproteinases (MMP-2, MMP-9) and cathepsin S with fragmentation of elastic lamellae and release of elastin-derived peptides, promoting osteochondrocytic vascular smooth muscle cell (VSMC) transdifferentiation [29-34]. The release of IL- 6 and TNF- $\alpha$, which are the first steps for activation of BMP2:BMP4, and Msx2, which promotes calcification by activating paracrine Wnt signals, and nuclear activation and localization of $\beta$-catenin, an indispensable coregulator of the expression of Runx2, osterix, Sox9, all transcription factors, are associated with osteochondrogenic phenotypic conversion of VSMC and pericytes [32-34]. Bone loss and osteolysis is a major complication of chronic inflammatory disease such as rheumatoid arthritis. TNF- $\alpha$ is a potent osteoclastogenic cytokine. It stimulates stromal cell production of IL-1, macrophage colony-stimulating factor, and receptor activator of NF-кB ligand (RANKL), which target macrophages to stimulate osteoclastic differentiation. RANKL is expressed in osteoblasts and activated T lymphocytes [35-37]. Normal bone remodeling is characterized by a balance between bone resorption by osteoclasts and bone matrix deposition by osteoblasts. This balance, which is disrupted in osteoporosis, is tightly regulated by the receptor activator of NF- $\mathrm{\kappa B}$ (RANK)/RANKL/osteoprote- gerin (OPG) signaling pathway [35-37]. RANKL is produced by osteoblasts and activates RANK expressed in osteoclasts and osteoclast receptors by nuclear translocation of an alternative NF- $\kappa \mathrm{B}$ pathway. RANK activation also suppresses osteoclast apoptosis. OPG is a decoy receptor expressed by osteoblasts, which binds to RANKL and directly controls RANKL-mediated actions. OPG also binds and neutralizes the proapoptotic actions of TNF-related apoptosis-inducing ligand [35-37]. Therefore, bone remodeling is critically dependent on the RANKL/OPG ratio with imbalance and an increase in this ratio leads to excessive bone resorption as observed in osteoporosis [38]. The discovery that OPG-deficient mice develop severe arterial calcifications in conjunction with severe osteoporosis, cortical and trabecular bone porosity, and high incidence of fractures provides robust evidence pointing out the RANK-RANKL-OPG system as a possible common factor linking osteoporosis and vascular calcifications [39]. The vascular calcification observed with RANKL/OPG was attributed to elevated bone resorption with excessive release of calcium and phosphates from the bone. A recent study has demonstrated that RANKL increases vascular smooth muscle cell calcification directly throughout activation of RANK expressed in VSMC and upregulation of BMP4 providing autocrine stimulus and activation of Wnt signaling [40]. OPG and its ligand RANKL are involved in immune-induced inflammatory responses including atherosclerosis. OPG is normally expressed in VSMC and endothelial cells where RANKL is usually absent. RANKL is expressed in inflammatory cytokine macrophages, endothelium and VSMC, and can directly induce calcification 
by BMP4 activation [40]. OPG may limit local inflammatory responses and 'neutralize' RANKL. In vitro OPG produced by smooth muscle and endothelial cells act as an antiapoptotic factor increasing endothelial cell survival [36]. Alterations of the RANKL/OPG ratio are critical in the evaluation of the clinical impact, and high OPG-associated cardiovascular risk could represent an inadequate response of OPG to increased RANKL activity [38].

While in the general population an association between osteoporosis and AC could be observed in the absence of overt mineral metabolism disorders, in CKD/ ESRD vascular calcifications and bone disorders are associated with deterioration of mineral and bone metabolism caused by changes in serum phosphorus and calci$\mathrm{um}$, and disruption of endocrine and humoral pathways including parathyroid hormone (PTH), calcitriol, FGF23/Klotho, and others [4, 5, 41]. Experimental and clinical data indicate that hyperphosphatemia plays a direct role in osteoblast-like transformation of VSMC by the upregulation of Runx $2 / \mathrm{Cbfa} 1$ and osterix transcription factors $[4,6]$.

In patients with $\mathrm{CKD} / \mathrm{ESRD}$, the association between bone and vascular calcifications concerns several aspects of bone disorders, i.e., high bone turnover (secondary hyperparathyroidism), low bone activity (adynamic bone disease), as well as low bone volume. In secondary hyperparathyroidism, the increased bone resorption associated with endogenous release of phosphate and calcium could play a critical role in the induction of vascular calcification. Chronic elevation of PTH also upregulates RANKL and downregulates OPG gene expression and enhances the RANKL/OPG ratio [42]. The biological alterations associated with chronic elevation of PTH differ from physiological, pulsatile variations of serum PTH. Indeed Shao et al. [43] demonstrated that daily injection of teriparatide (human parathyroid hormone 1-34) inhibits osteogenic vascular calcification in diabetic low-density lipoprotein receptor-deficient mice. Using the same animal model, Cheng et al. [44] demonstrated that activation of vascular smooth muscle parathyroid hormone receptor inhibits Wnt/ $\beta$-catenin signaling, which is essential for Runx 2 expression and osteogenic orientation of mesenchymal cells.

In CKD/ESRD patients, arterial and valvular calcifications are more frequently observed in the presence of low bone turnover disease with a decreased osteoblast number or osteoblastic activity suggesting that osteoblasts could directly or indirectly influence vascular function and calcification.

\section{Bone as the 'Culprit' of Arterial Alterations?}

Classically known as a system providing support for locomotion and mineral homeostasis, the bone has metabolic functions interacting with whole body physiology. Several experimental studies have shown that osteoblasts are involved in the control of fat tissue metabolism and adipokine release, energy expenditure, insulin secretion and sensitivity, all factors that have a direct influence on cardiovascular function and health. Lee et al. [45] showed that osteoblasts exert an endocrine regulation on energy metabolism with osteocalcin playing an important role. Uncarboxylated osteocalcin can regulate the expression of insulin genes and $\beta$-cell proliferation, adiponectin release and expression in adipocytes [46, 47]. In the general population and in CKD patients, serum osteocalcin was positively associated with adiponectin $[47,48]$. Adiponectin protects arteries against hypertension, suppresses atherosclerosis and activates osteoblastogenesis $[49,50]$. An inverse relationship was observed between adiponectin and osteocalcin and arterial stiffness and progression of coronary calcifications [47]. Reduced plasma levels of adiponectin are found in metabolic syndrome and type 2 diabetes. Multiple hormones involved in endocrine regulation of adipose tissue and energy metabolism could affect bone structure, including leptin. Leptin is a powerful inhibitor of bone formation in vivo [51] and favors vascular calcification [52]. In ESRD, serum leptin is increased and associated with low PTH [53], suggesting that in ESRD leptin might reduce bone activity and promote arterial calcifications.

In conclusion, an increased number of publications on subjects from the general population and CKD/ESRD patients show a significant association between arterial pathology (atherosclerosis and arterial calcifications) and bone disorder including osteoporosis, and high or low bone activity. The pathophysiology and biological links between bone and arterial abnormalities suggest the existence of a bone-vascular cross-talk. The nature of this is not well understood: it could result from (a) action of common factors on bone remodeling and atherosclerosis/ calcification; (b) direct action of bone cells (osteoblasts/ osteocytes) on vascular biology and structure, or (c) compromised bone blood supply related to arteriosclerosis of bone vessels and reduced perfusion. 


\section{References}

$>1$ Braun J, Oldendorf M, Moshage W, et al: Electron-beam computed tomography in the evaluation of cardiac calcifications in chronic dialysis patients. Am J Kidney Dis 1996;27: 394-401.

-2 London GM, Guérin AP, Marchais SJ, et al: Arterial media calcification in end-stage renal disease: impact on all-cause and cardiovascular mortality. Nephrol Dial Transplant 2003;18:1731-1740.

>3 Guérin AP, London GM, Marchais SJ, Métivier F: Arterial stiffening and vascular calcifications in end-stage renal disease. Nephrol Dial Transplant 2000;15:1014-1021.

-4 Schoppet M, Shroff RC, Hofbauer LC, Shanahan CM: Exploring the biology of vascular calcification in chronic kidney disease: what's circulating? Kidney Int 2008;73:384390.

$>5$ Demer LL, Tintut Y: Vascular calcification: pathobiology of multifaceted disease. Circulation 2008;117:2938-2948.

-6 Reynolds JL, Joannides AJ, Skepper JN, et al: Human vascular smooth muscle cells undergo vesicle-mediated calcification in response to changes in extracellular calcium and phosphate concentrations: a potential mechanism for accelerated vascular calcification in ESRD. J Am Soc Nephrol 2004;15:28572867.

7 Schulz E, Arfai K, Liu X, et al: Aortic calcification and the risk of osteoporosis and fractures. J Clin Endocrin Metab 2004;89:42464253.

$>8$ Hamerman D: Osteoporosis and atherosclerosis: biological linkage and the emergence of dual-purpose therapies. Q J Med 2005;98: 467-484.

9 Tanko LB, Christiansen C, Cox DA, et al: Relationship between osteoporosis and cardiovascular disease in postmenopausal women. J Bone Mineral Res 2005;20:1912-1920.

10 Sumino H, Ichikawa S, Kasama S, et al: Elevated arterial stiffness in postmenopausal women with osteoporosis. Maturitas 2006; 55:212-218

11 Barreto DV, Barreto FC, Carvalho AB, Cuppari L, Cendoroglo M, Draibe SA, Moyses RM, Neves KR, Jorgetti V, Blair A, Guiberteau R, Fernandes Canziani ME: Coronary calcification in hemodialysis patients: the contribution of traditional and uremia-related risk factors. Kidney Int 2005;67:15761582 .

-12 London GM, Marty C, Marchais SJ, Guerin AP, Metivier F, de Vernejoul MC: Arterial calcifications and bone histomorphometry in end-stage renal disease. J Am Soc Nephrol 2004;15:1943-1951.

$\checkmark 13$ Adragao T, Herberth J, Monier-Faugere $\mathrm{M}-\mathrm{C}$, et al: Low bone volume - a risk factor for coronary calcifications in hemodialysis patients. Clin J Am Soc Nephrol 2009;4:450455 .
14 London GM, Marchais SJ, Guérin AP, et al: Association of bone activity, calcium load, aortic stiffness, and calcifications in ESRD. J Am Soc Nephrol 2008;19:1827-1835.

15 Joki N, Hase H, Shiratake M, et al: Calcaneal osteopenia is a new marker for arterial stiffness in chronic hemodialysis patients. Am J Nephrol 2005;25:196-202.

-16 Raggi P, Bellasi A, Ferramosca E, et al: Pulse wave velocity is inversely related to vertebral bone density in hemodialysis patients. Hypertension 2007;49:1278-1284.

17 Toussaint ND, Lau KK, Strauss BJ, et al: Association between vascular calcification, arterial stiffness and bone mineral density in chronic kidney disease. Nephrol Dial Transplant 2008;23:586-593.

18 Bridgeman G, Brookes M: Blood supply to the human femoral diaphysis in youth and senescense. J Anat 1996;188:611-621.

19 Ramseier E: Untersuchungen über arteriosklerotische Veränderungen der Knochenarterien. Wirchow Arch Path Anat 1962;336: 77-86.

20 Alagiakrishnan K, Juby A, Hanley D, et al: Role of vascular factors in osteoporosis. J Gerontol 2008;4:362-366.

21 Griffith JF, Yeung DK, Tsang PH, et al: Compromised bone marrow perfusion in osteoporosis. J Bone Miner Res 2008;23:10681075.

22 Laroche M: Intraosseous circulation from physiology to disease. Joint Bone Spine 2002; 69:262-269.

23 Parhami F, Garfinkel A, Demer LL: Role of lipids in osteoporosis. Arterioscler Thromb Vasc Biol 2000;20:2346-2348.

24 Koh JM, Khang YH, Jung CH, Bae S, Kim DJ, Chung YE, Kim GS: Higher circulating hsCRP levels are associated with lower bone mineral density in healthy pre- and postmenopausal women: evidence for a link between systemic inflammation and osteoporosis. Osteoporos Int 2005;16:1263-1271.

25 Gerdhem P, Obrant KJ: Effects of cigarette smoking on bone mass as assessed by dualenergy X-ray absorptiometry and ultrasound. Osteoporos Int 2002;13:932-936.

26 Mody N, Parhami F, Sarafian TA, Demer LL: Oxidative stress modulates osteoblastic differentiation of vascular and bone cells. Free Radic Biol Med 2001;31:509-519.

27 Tanikawa T, Okada Y, Tanikawa R, Tanaka Y: Advanced glycation end-products induce calcification of vascular smooth muscle cell throughout RAGE/p38MAPK. J Vasc Res 2009;46:572-580.

28 Hjortnaes J, Butcher J, Figueiredo J-L, et al: Arterial and aortic valve calcification inversely correlates with osteoporotic bone remodelling: a role of inflammation. Eur Heart J 2010;31:1975-1984.
29 Aikawa E, Nahrendorf N, Figueiredo J-L, et al: Osteogenesis associates with inflammation in early-stage atherosclerosis evaluated by molecular imaging in vivo. Circulation 2007;116:2841-2850.

30 Deguchi J-O, Aikawa M, Tung C-H, et al: Inflammation in atherosclerosis: visualizing matrix metalloproteinase action in macrophages in vivo. Circulation 2006;114:55-62.

31 Aikawa E, Nahrendorf M, Sosnovik D, et al: Multimodality molecular imaging identifies proteolytic and osteogenic activities in early aortic valve disease. Circulation 2007;115: 377-386.

-32 Aikawa E, Aikawa M, Libby P, et al: Arterial and aortic valve calcification abolished by elastolytic cathepsin S deficiency in chronic renal disease. Circulation 2009;119:17851794.

33 Al-Aly Z, Shao J-S, Lai Ch-T, et al: Aortic Msx2-Wnt calcification cascade is regulated by $\mathrm{TNF}-\alpha$-dependent signal in diabetic $\mathrm{Ldlr}^{-1-}$ mice. Arterioscler Thromb Vasc Biol 2007;27:2589-2596.

34 Shao J-S, Al-Aly Z, Lai Ch-T, et al: Vascular Bmp-Msx2-Wnt signaling and oxidative stress in arterial calcification. Ann NY Acad Sci 2007;1117:40-50

35 Hofbauer LC, Schoppet M: Clinical implications of the osteoprotegerin/RANKL/RANK system for bone and vascular diseases. JAMA 2004;292:490-495.

>36 Collin-Osdoby P: Regulation of vascular calcification by osteoclast regulatory factors RANKL and osteoprotegerin. Circ Res 2004; 95:1046-1057.

37 Teitelbaum SL: Osteoclasts; culprit in inflammatory osteolysis. Arthritis Res Ther 2006;8:1-8

38 Flore CE, Pennisi P, Ferro G, et al: Altered osteoprotegerin/RANKL ratio and low bone mineral density in celiac patients on longterm treatment with gluten-free diet. Horm Metab Res 2006;38:417-422.

39 Bucay N, Sarosi I, Dunstan CR, et al: Osteoprotegerin-deficient mice develop early onset osteoporosis and arterial calcification. Genes Dev 1998;12:1260-1268.

40 Panizo S, Cardus A, Encinas M, et al: RANKL increases vascular smooth muscle cell calcification through a RANK-BMP4-dependent pathway. Circ Res 2009;104:1041-1048.

-41 Moe S, Drüeke T, Cunningham J, et al: Definition, evaluation, and classification of renal osteodystrophy: a position statement from Kidney Disease: Improving Global Outcomes (KDIGO). Kidney Int 2006;69:19451953.

42 Huang JC, Sakata T, Pfleger LL, et al: PTH differentially regulates expression of RANKL and OPG. J Bone Miner Res 2004; 19:234-244. 
43 Shao J-S, Cheng S-L, Charlton-Kachigian N, et al: Teriparatide (human parathyroid hormone (1-34)) inhibits osteogenic vascular calcification in diabetic low density lipoprotein recepto-deficient mice. J Biochem Chemistry 2003;278:50195-50202.

44 Cheng S-L, Shao J-S, Halstead LR, et al: Activation of vascular smooth muscle parathyroid receptor inhibits Wnt/ $\beta$-catenin signaling and aortic fibrosis in diabetic arteriosclerosis. Circ Res 2010;107:271-282.

45 Lee NK, Sowa H, Hinoi E, et al: Endocrine regulation of energy metabolism by the skeleton. Cell 2007;130:456-469.
46 Ferron M, Hinoi E, Karsenty G, Ducy P: Osteocalcin differentially regulates $B$ cells and adipocytes gene expression and affects the development of metabolic disease in wildtype mice. Proc Natl Acad Sci USA 2008; 105 5266-5270.

47 Kanazawa I, Yamaguchi T, Yamamoto M, et al: Serum osteocalcin is associated with glucose metabolism and atherosclerosis parameters in type 2 diabetes mellitus. J Clin Endocrinol Metab 2009;94:45-49.

48 Bacchetta J, Boutroy S, Guebre-Egzlabher F, et al: The relationship between adipokines, osteocalcin and bone quality in chronic kidney disease. Nephrol Dial Transplant 2009; 24:3120-3125.

49 Oshima K, Nampei A, Matsuda M, et al: Adiponectin increases bone mass by suppressing osteoclasts and activating osteoblasts. Biochem Biophys Res Commun 2005;331: 520-526.
50 Luo XH, Guo LJ, Yuan LQ, et al: Adiponectin stimulates human osteoblasts proliferation and differentiation via the MAPK signaling pathway. Exp Cell Res 2005;309:99-109.

51 Elefteriou F, Takeda S, Ebihara K, et al: Serum leptin level is a regulator of bone mass. Proc Natl Acad Sci USA 2004;101:32583263.

52 Parhami F, Tintut Y, Ballard A, et al: Leptin enhances the calcification of vascular cells: artery wall as a target of leptin. Circ Res 2001; 88:954-960.

53 Coen G, Ballanti P, Fischer MS, et al: Serum leptin in dialysis renal osteodystrophy. Am J Kidney Dis 2003;42:1036-1042. 